\title{
Experiencias en la aplicación del Pile Integrity Tester en el Control de la integridad de pilotes hormigonados en el lugar (in situ) pertenecientes a la cimentación del Proyecto TP-Sector Oasis, Varadero
}

\author{
ing. H. Rodríguez ${ }^{1 *}$, Dr. C. M. Aguilar ${ }^{2 *}$ \\ Autor de contacto: * henryrc@eniahab.cu * manuelab@eniahab.cu \\ ${ }^{1}$ Departamento de Patología y Diagnóstico de estructuras de hormigón, ENIA-INVESCONS, La Habana, Cuba \\ ${ }^{2}$ Departamento de Patología y Diagnóstico de estructuras de hormigón, ENIA-INVESCONS, La Habana, Cuba
}

\section{Resumen}

En el presente trabajo se abordan las experiencias en el control de la integridad de pilotes fundidos en el lugar (drilled shaft pile), para la cimentación del Proyecto TP Sector Oasis, Varadero, Cuba, usando el Pile Integrity Tester, de la compañía Pile Dynamics Inc.

Los pilotes fundidos en el lugar brindan estabilidad a estructuras complejas en condiciones geotécnicas especiales, pero, una vez construidos, resulta difícil evaluar su continuidad geométrica, debido a su inaccesibilidad, quedando enterrada en el suelo. Con la finalidad de detectar estos defectos, fallos o discontinuidades, se llevó a cabo una campaña de ensayos PIT al total de los pilotes excavados y fundidos de la cimentación; actividad factible, debido a su rapidez de ejecución y bajo costo.

Palabras claves: Pile Integrity Tester; integridad de pilotes fundidos en el lugar; defectos, fallos o discontinuidades. 


\begin{abstract}
In the present work are addressed the experiences in the control of the integrity of drilled shaft piles, for the foundation of the TP Sector Oasis Project, Varadero, Cuba, using the Pile Integrity Tester, from the Pile Dynamics Company. Inc.

Drilled shafts piles provide stability to complex structures under special geotechnical conditions, but once built, it is difficult to assess their geometric continuity, due to their inaccessibility, remaining buried in the ground. In order to detect these defects, failures or discontinuities, a PIT test campaign was carried out on all the excavated and cast piles of the foundation, feasible activity, due to its speed of execution and low cost.
\end{abstract}

Keywords: Pile Integrity Tester; integrity of drilled shaft pile; defects, failures or discontinuities.

\title{
Resumo
}

Neste trabalho, são abordadas experiências no controle da integridade de estacas lançadas no local (estaca furada), para a fundação do Projeto Oásis do Setor TP, Varadero, Cuba, utilizando o testador de integridade de pilha, da empresa Pile Dynamics. Inc.

As estacas moldadas no local proporcionam estabilidade a estruturas complexas em condições geotécnicas especiais, mas uma vez construídas, é difícil avaliar sua continuidade geométrica, devido à sua inacessibilidade, permanecendo enterradas no solo. Para detectar esses defeitos, falhas ou descontinuidades, foi realizada uma campanha de PIT teste em todas as estacas escavadas e lançadas da fundação; atividade viável, devido à sua rapidez de execução e baixo custo.

Palavras-chave: Pile Integrity Tester; integridade das estacas fundidas no local; defeitos, falhas ou descontinuidades.

\section{Introducción}

Los ensayos no destructivos o no invasivos se abren paso cada vez más imponente en el ámbito de la exploración ingenieril, como herramientas eficaces en el control de la integridad de estructuras como pilotes, aprovechando el avance de las ciencias informáticas al unísono del acelerado desarrollo de la tecnología, estos ensayos son de rápida aplicación $\mathrm{y}$ muy factibles económicamente.

El ensayo de Integridad empleando el Método Sónico o Sonic Echo permite determinar la presencia de defectos constructivos en elementos estructurales axiales como pilotes, columnas y vigas. El Pile Integrity Tester (PIT) figura como una de las herramientas más usadas en el mundo de la exploración de estructuras, aunque no brinda información sobre capacidad de los pilotes y no es posible sustituir sus resultados por los obtenidos en una Prueba de Carga.

La metodología y principios del ensayo, se encuentran recogidos en la (ASTM D 5882, 2000. Standard test method for low strain integrity testing of piles. American Society for Testing and Materials).

El PIT ha sido aplicado en países como España, México, EUA, Canadá, Colombia, Chile, y otros. 
En este trabajo, se muestran los resultados de una campaña de 7 meses de investigación con un promedio de 50 pilotes ensayados diariamente en el Proyecto Sector TP Oasis de la Playa de Varadero, Cuba, (Figura 1) utilizando el PIT en provecho de los principios de la ecuación de onda. En TP Sector Oasis, se proyectaron 1033 pilotes hormigonados in situ que trabajarían en punta y a fricción, como elementos de cimentación, de estos, se le ejecutó el ensayo de integridad a 1007, obteniéndose al menos, 4 registros de ensayo por cada uno. Para la interpretación de los resultados, se tomó en cuenta el estudio geotécnico, la composición geológica del área y las condiciones finales de instalación de los pilotes, información recogida en reportes elaborados por Bauer Fundaciones, corporativa latinoamericana de la Empresa germánica que llevó a cabo la perforación de los pilotes. Además, se incorporó, al análisis del ensayo, toda la información disponible del proyecto de ejecución: el diseño geotécnico, y el proceso de construcción de estos, la excavación, colocación del acero de refuerzo y el hormigonado.

Las modernas técnicas electrónicas e informáticas permitieron un procesamiento y un tratamiento de las señales obtenidas que facilitó la posterior interpretación y presentación de los resultados.

\section{Planteamiento de la Metodología de Investigación Científica Situación problémica}

La geometría de los pilotes de hormigón fundidos in situ que constituyen la cimentación de los edificios proyectados (Dos Hoteles y un Centro Comercial) en TP Sector Oasis, Varadero, ha quedado enterrada en las capas ingenieriles del subsuelo, una vez constituido el nivel de solera, quedando ocultos posibles defectos o discontinuidades en el hormigón que pudieran provocar fallos estructurales o mal funcionamiento de estos, como elementos de entrega de cargas al terreno, por lo que se necesita un método que permita obtener información de toda la longitud de sus cuerpos desde la cabeza hasta la punta, para de esta manera auxiliar el criterio de aceptación o descarte de un alguno de estos elementos para cumplir su función estructural.

\section{Hipótesis}

Si es posible utilizar una herramienta fundamentada científicamente, mediante la cual podamos obtener información del volumen de los pilotes en profundidad, será posible:

1-Localizar discontinuidades, defectos o cualquier otro signo desfavorable a lo largo del fuste que comprometa el mal funcionamiento de los pilotes como estructuras de transferencia de cargas.

2-Corregir el diseño geotécnico de los pilotes

3-Comprobar que la geometría de los pilotes cumpla con las tolerancias diseñadas, dicho de otra manera, que no ocurran estrangulamientos o ensanchamientos de sección que influyan en el funcionamiento geotécnico o bien que exista pilote en toda su longitud.

\section{Objetivo del ensayo}

Conocer con buen nivel de aproximación el estado contextual del pilote, dígase, su continuidad estructural, longitud y volumen, una vez que han quedado enterrados. 


\section{Método}

Una campaña de ensayos sónicos de integridad de los pilotes, como método de exploración indirecta, usando el PIT, permitirá entre otras funciones, evaluar la uniformidad y la continuidad del hormigón en profundidad y localizar defectos que pudieran generar fallos o discontinuidades en el hormigón que invaliden a los pilotes como aptos para cumplir su función estructural

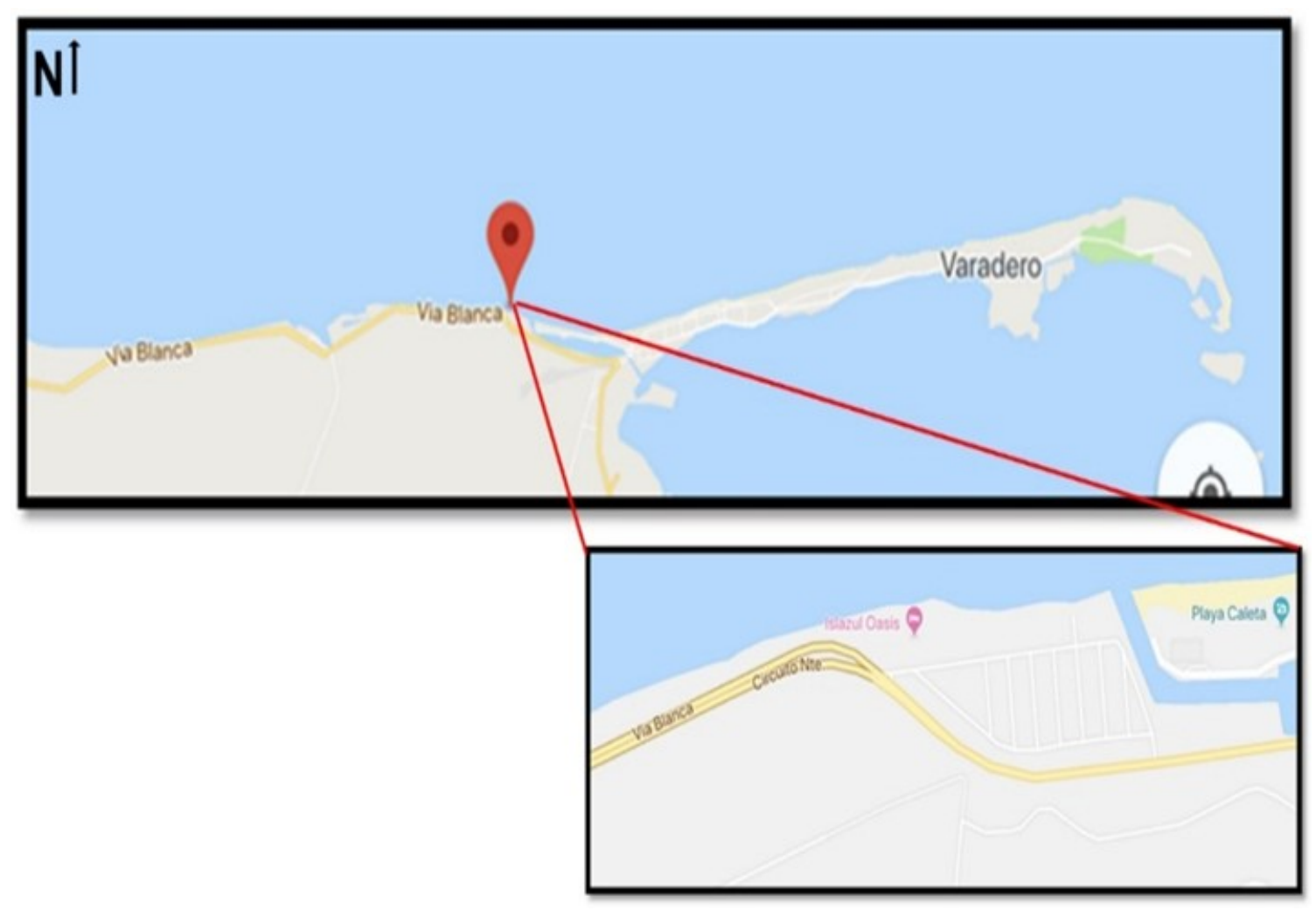

Figura 1. Situación Obra TP-Sector Oasis. Autopista Sur Vía Blanca, Varadero, Matanzas, Cuba. Aplicación de ensayos dinámicos sónicos in situ (PIT) a la integridad del hormigón de cimentaciones profundas (pilotes)

\section{Fundamentos teóricos y principio de aplicación del ensayo de integridad de pilotes}

El ensayo de integridad con el PIT se basa en el principio teórico de la propagación de ondas longitudinales de baja deformación que genera efectos de dilatación y compresión a lo largo del fuste del pilote (Colectivo de Autores Pile Dynamics Inc., 2014). La velocidad de propagación de la onda es directamente proporcional al módulo de elasticidad e inversamente proporcional a la densidad del material. El frente de onda que se genera en el punto de impacto, se puede considerar como un frente de onda plano que se transmite por el cilindro (forma geométrica del pilote) a una velocidad:

$c=\sqrt{E / \rho}$ 
c: velocidad de propagación de la onda comprimida

E: módulo de elasticidad dinámico del material constituyente del pilote (hormigón)

$\rho$ : densidad de masa del material constituyente del pilote (hormigón)

La onda es reflejada por las discontinuidades del pilote, por la punta del pilote, por cambios de sección o por las características típicas del terreno donde se ha instalado (Fundaciones, L. d. (s.f.). Protocolo de ensayo de integridad de pilotes). La cantidad de energía que se transmite al terreno que rodea al pilote dependerá de la relación entre las rigideces del pilote y del terreno. Si el suelo que rodea al pilote es blando o suelto, como suele ocurrir en las condiciones en las que se cimienta con pilotes, una parte importante de la energía del tren de ondas se transmite a lo largo del pilote hasta que se disipa totalmente o encuentra un cambio geométrico o de material en el cilindro.

Cuando hay cambio geométrico o de características del material de que se constituye el pilote o un cambio importante de la rigidez del terreno, parte de la energía se refleja hacia la cabeza de éste. El método de ensayo se basa en el análisis de la onda que es reflejada hacia la cabeza del pilote.

Los cambios en las características geométricas o en las propiedades del material que lo constituyen y que producen reflexiones que permiten diagnosticar el estado del pilote son:

- el área de la sección transversal del pilote,

- densidad del material y/o módulo de elasticidad

- velocidad de transmisión de la onda en el material. (hormigón)

Estos parámetros tributan al Principio de la Impedancia Acústica y se relacionan mediante la ecuación:

$z=\rho \cdot c \cdot A$

z: impedancia de una sección del pilote

$\rho$ : densidad del material

c: velocidad de propagación de la onda de compresión

A: área de la sección transversal del fuste

La onda es captada por un acelerómetro conectado al equipo PIT, que amplifica y digitaliza la señal mediante un sistema electrónico y se convierte en medida de velocidad (Dominio espacial del tiempo), que se presenta inmediatamente en la pantalla del equipo para comprobar la correcta ejecución del ensayo, quedando registrada, para su posterior interpretación en gabinete.

\section{Accesorios para el Ensayo de Integridad}

Dispositivo Pile Integrity Tester (PIT). (Figura 2 A)

$>$ Martillo convencional cableado de $53.4 \mathrm{~kg}$ (Figura 2 B-1) (prueba de fuerza, trabaja en el dominio de la frecuencia mediante transformación matemática por series rápidas de Fourier). 
Martillo convencional portátil de $4.5 \mathrm{~kg}$ (Figura $2 \mathrm{~B}-2$ ) (prueba de velocidad, trabaja en el dominio espacial del tiempo, conocida la velocidad, puede calcularse la longitud del pilote, muy efectivo para pilotes de poca esbeltez).

$>$ Acelerómetro convencional cableado (Figura 2 B-4) (se conecta a la unidad velocidad (V), si evaluamos el dominio tiempo, mientras que, si medimos fuerza sobre la cabeza del pilote, se conecta el martillo de 53,4 kg a la unidad Fuerza (F)

$>$ Otros dispositivos del modelo inalámbrico

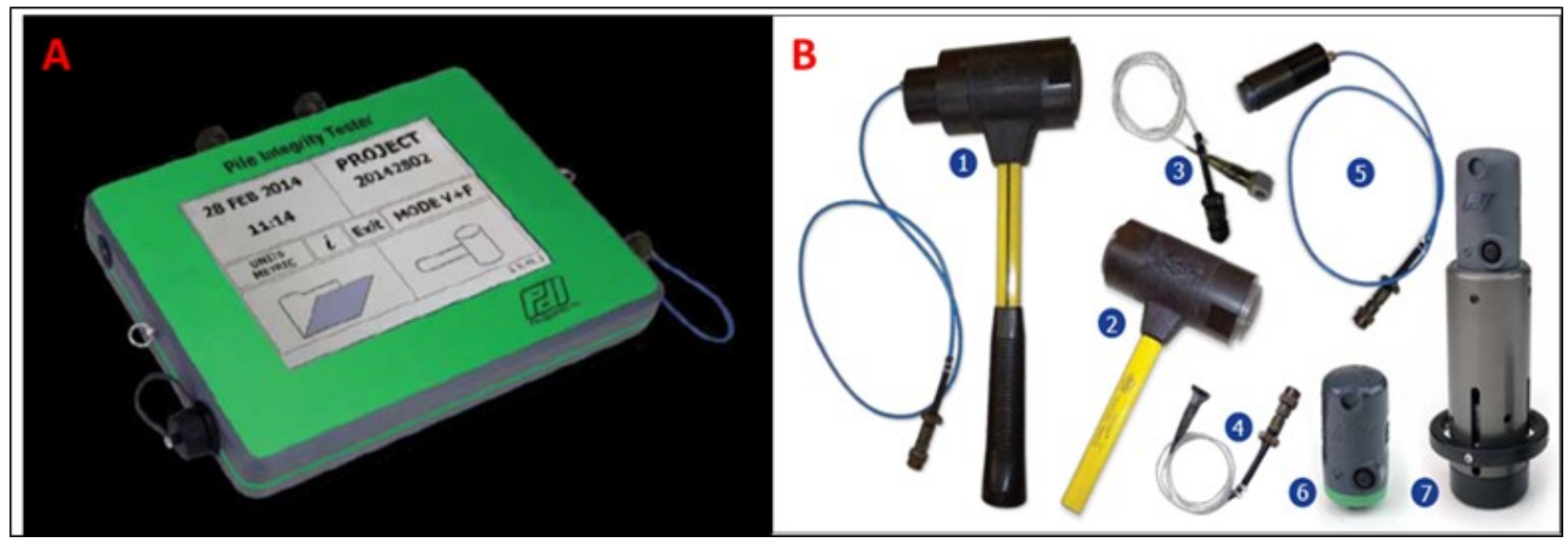

Figura 2. A. Dispositivo PIT e interfaz con menú principal. B. Accesorios para la ejecución de los ensayos. Fuente: Manual PIT.

\section{Preparación de los pilotes para el ensayo PIT}

La edad mínima del hormigón en el momento del ensayo es de siete días, para garantizar un grado de endurecimiento y un módulo de elasticidad que permitan que la onda se propague.

\section{Metodología de ejecución del ensayo en el terreno}

Para asegurar una correcta ejecución de la prueba se deben tener en cuenta los siguientes aspectos:

a) Asegurar que la edad mínima del concreto de los pilotes a ensayar sea de siete (7) días o cuando el concreto alcance el $75 \%$ de la resistencia de diseño.

b) La superficie superior del pilote debe estar seca, horizontal y lisa, si existe algún "solado" se debe dilatar respecto al concreto del pilote (Santos, Y., 2016). (Figura 3 D)

c) Si existe en algún elemento concreto de "limpia" para el armado del cabezal, este debe ser dilatado respecto a los pilotes a ensayar.

d) El equipo de prueba de integridad debe estar expuesta a la menor cantidad de sonidos con el fin de obtener una alta resolución, en las colindancias al ensayo no deben estar funcionando maquinarias o motores que contaminen con señales de ruido el ensayo.

e) Se solicita el registro de excavación y hormigonado de los pilotes ensayados avalados por la interventoría de la obra, al igual que los datos de geometría, resistencia, volúmenes teóricos y reales de concreto, tiempo de llegada y descarga del hormigón.

Una vez se ha preparado el pilote para ser ensayado, se limpia con una escobilla de mano y se coloca el acelerómetro sobre una superficie horizontal de la cabeza del pilote, procurando que quede lo más vertical posible, y comprobamos con el martillo que sea una zona compacta de 
hormigón. Los golpes deben ser suaves y secos, y preferentemente siempre en el mismo lugar. Cada golpe de martillo, nos representa una curva en el reflectograma (Maestre Mesa y Rodríguez Cárdenas, (2019).

Una vez obtenidas graficas válidas, procedemos a la conservación de la información para su posterior interpretación.
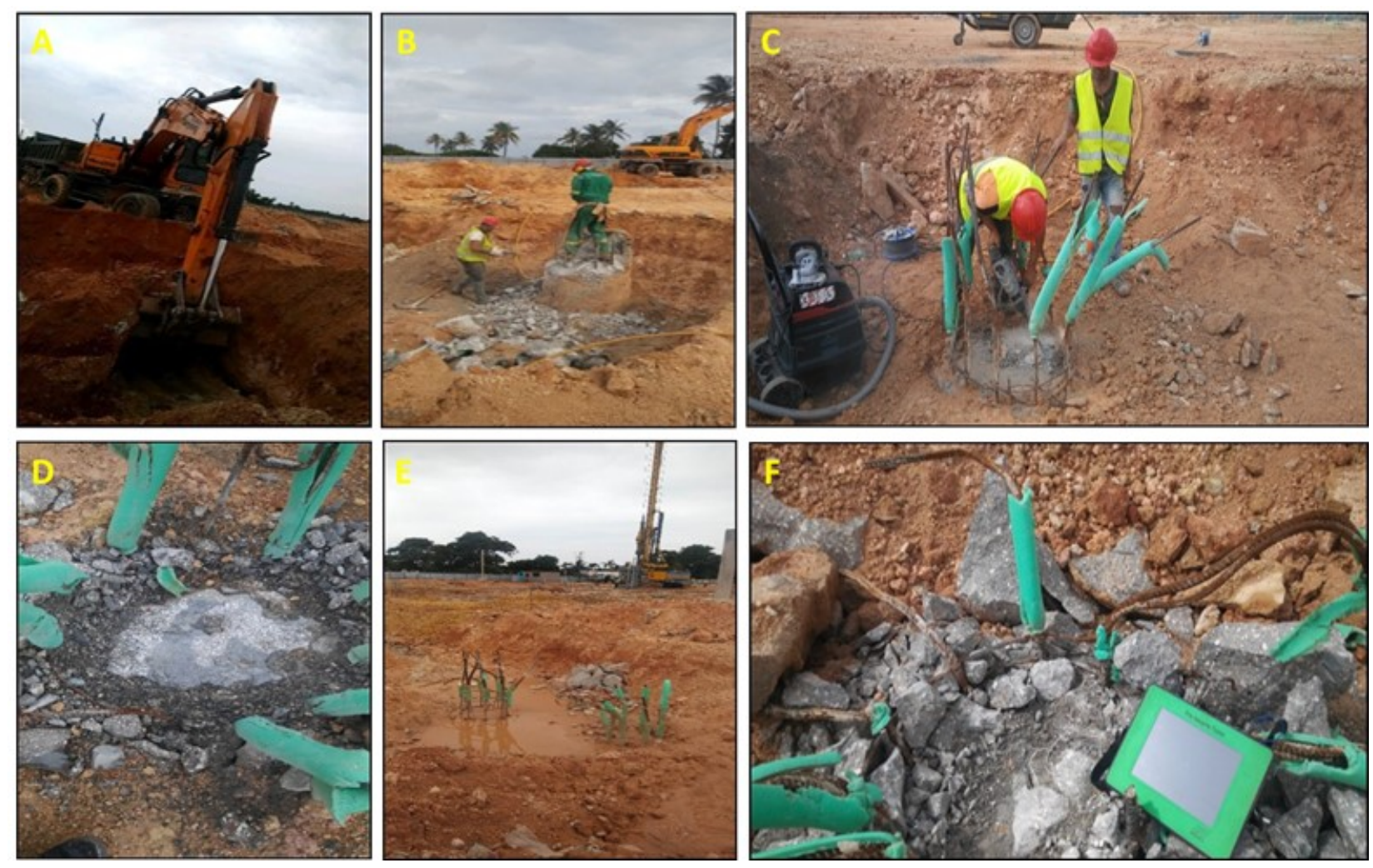

Figura 3. Procesos de preparación de la cabeza del pilote para ensayos de integridad. factores que obstaculizan el proceso de ensayo. A. Descubrimiento del pilote. B. descabezado con martillo hidráulico. C. Planeado de la superficie de la cabeza del pilote. D. Segregación de hormigón en la cabeza del pilote. E. Presencia de agua en la superficie, humedad permanente. $F$. Irregularidades en la superficie de la cabeza por Planeado incorrecto

\section{Clasificación del tipo de registro según la morfología del reflectograma}

Registros AA: Pilote sano. Los pilotes que presentan reflexiones insignificantes de la onda sónica en puntos del fuste del pilote por encima de la punta. Puede ser identificada una reflexión clara de la punta del pilote correspondiente a la longitud registrada y una velocidad de onda dentro de un rango aceptable. Registros de esta categoría deben indicar normalmente variaciones aceptadas del tamaño y calidad del material.

Registros AB, ABx: Cuando no se aprecia una reflexión clara de la onda sónica en la punta, ni aparecen reflexiones que indiquen una reducción de la sección del pilote o mala calidad del material, aunque tampoco existe una evidencia positiva de que el pilote esté libre de defectos. 
Registros ABx: En el caso ABx se establece hasta que profundidad el pilote puede considerarse libre de defecto. Este tipo de registro es frecuente en el caso de pilotes muy esbeltos instalados en suelos de alta resistencia.

Registros PFx: Existe una anomalía que indica un probable fallo a una profundidad x y existe evidencia de una reflexión correspondiente a la punta del pilote. En aquellos casos en que se apreciaron reflexiones significativas o anomalías de la onda por encima de la punta del pilote, se da una interpretación evaluando los posibles fallos en el pilote.

Registros PDx: El registro muestra una reflexión fuerte correspondiente a una reducción importante de la sección del pilote o la calidad del material y no existe evidencia de la reflexión procedente de la punta del pilote.

Registros IVx: Registros inciertos por debajo de una profundidad x, afectados por vibraciones generadas por maquinaria de construcción o refuerzo muy denso sobre la cabeza del pilote, que no permiten llegar a una conclusión clara sobre la integridad del pilote ensayado.

Registros IR: Registros inciertos; los datos son inciertos, posiblemente por razones tales como:

- Mala calidad del punto de impacto y registro en la cabeza del pilote por baja resistencia del hormigón, discontinuidades, irregularidades, etc.

- Cambios de impedancia planificados por uniones las cuales generan señales que impiden la identificación de la señal de la punta.

\section{Aplicación de ensayos de integridad (PIT) a pilotes del Proyecto TP Sector Oasis, Varadero}

\section{Caracterización geológica del subsuelo donde se fundieron los pilotes}

La investigación geotécnica fue desarrollada por especialistas de la ENIA, así como la descripción del corte geológico.

Hacia arriba, en el corte de la parcela, se presenta una roca carbonatada organógena, con abundante aparición de corales, de alta compacidad y resistencia, con desarrollo de carso, perteneciente posiblemente a la Fm Canímar (Capa E). El techo de esta capa se encuentra a profundidades variables, pero queda bien esclarecido que hacia el área que ocupa la cimentación del hotel H2, aparece a una menor profundidad con respecto al área que ocupa la cimentación del hotel H1, por lo que un gran número de pilotes de $\mathrm{H} 2$ quedaron apoyados en esta capa, no así en $\mathrm{H} 1$. Así pues, en $\mathrm{H} 2$, los pilotes superiores a 15 metros se apoyan todos en esta secuencia, mientras que en H1 los pilotes de similar longitud generalmente no llegan hasta ella en varias zonas.

Sobre esta secuencia, aparece, con mayor presencia hacia H2, un horizonte de material arcilloarenoso del orden desde algunos centímetros hasta el metro e incluso 2 metros, esporádicamente. Esta delgada capa proviene de la acumulación de los materiales de la erosión de la citada Fm Canímar. El comportamiento de este horizonte parece ser inestable, poco compacto y afectado por la alteración físico-química.

La Capa D se encuentra sobreyaciendo discordantemente a este horizonte; Se describen como rocas terrígeno-carbonatadas, posiblemente, calcarenitas, muy alteradas, con alternancias de capas arenoarcillosas, perteneciente a la Formación Jaimanitas, constituyendo su base. El contacto de la capa $\mathrm{D}$ con el material areno arcilloso descrito anteriormente, como promedio a unos 14 metros de profundidad con respecto al nivel de terreno, en el caso de H2. En H1 se encuentra a una mayor profundidad. 
En H2 por encima de este paquete terrígeno-carbonatado aparece siempre la capa $\mathrm{C}$ que es un bloque carbonatado de poco espesor y alta estabilidad y dureza.

\section{Perforación y hormigonado de los pilotes}

El servicio de construcción de los pilotes lo ejecutó la empresa Bauer Fundaciones América Latina S.A., con un equipo compuesto por dos máquinas perforadoras Bauer BG-28, dos ingenieros civiles, dos operarios, dos jefes de cuadrilla y ayudantes.

Las incidencias y detalles del proceso de perforación y hormigonado de los pilotes se registraron en detallados reportes donde se encuentra información necesaria para verificar las condiciones de instalación de cada pilote.

Durante todo el proceso de construcción se registraron importantes sobreconsumos de hormigón con respecto al previsto en el proyecto preliminar de Bauer. En los reportes se registran dichos sobreconsumos y de las curvas de hormigonado se puede diferenciar el hormigón empleado en rellenar cavernas y oquedades del hormigón empleado en rellenar e incrementar la sección de los pilotes (sobredimensionamiento).

En pilotes donde ocurrió estrangulamiento de una sección del fuste, debido a la interacción suelopilote, se consideró el sobrediseño de estos elementos, no comprometiendo nunca su función estructural.

\section{Ensayo Sónico de Integridad de Pilotes (PIT). Resultados e Interpretación}

Se ejecutaron ensayos a 1017 pilotes (Figura 4). A cada pilote se le tomó un mínimo de 4 puntos de ensayo para obtener información representativa de todo el volumen de hormigón del pilote (Figura 5).

El descabezado del pilote se ejecutó usando el método llamado recipieaux o demolición con martillo hidráulico, neumático y eléctrico. El recipieaux consiste en verter en la sección del pilote seleccionada un líquido expansivo que al fraguar y expandirse crea una superficie de falla de la cual puede desprenderse la sección del pilote prevista. Dicho método se les aplicó a todos los pilotes, pero sólo se obtuvo resultados en una pequeña parte de ellos. En los registros PIT generalmente, se podían establecer diferencias entre los pilotes descabezados manualmente y los descabezados por recipieaux.

El descabezado por demolición con martillo fue el más utilizado y generalmente produjo agrietamiento en la cabeza del pilote, que en muchos casos afectó la calidad de los registros PIT. A continuación, se presentan a manera de ejemplo algunos registros representativos con los comentarios correspondientes: 


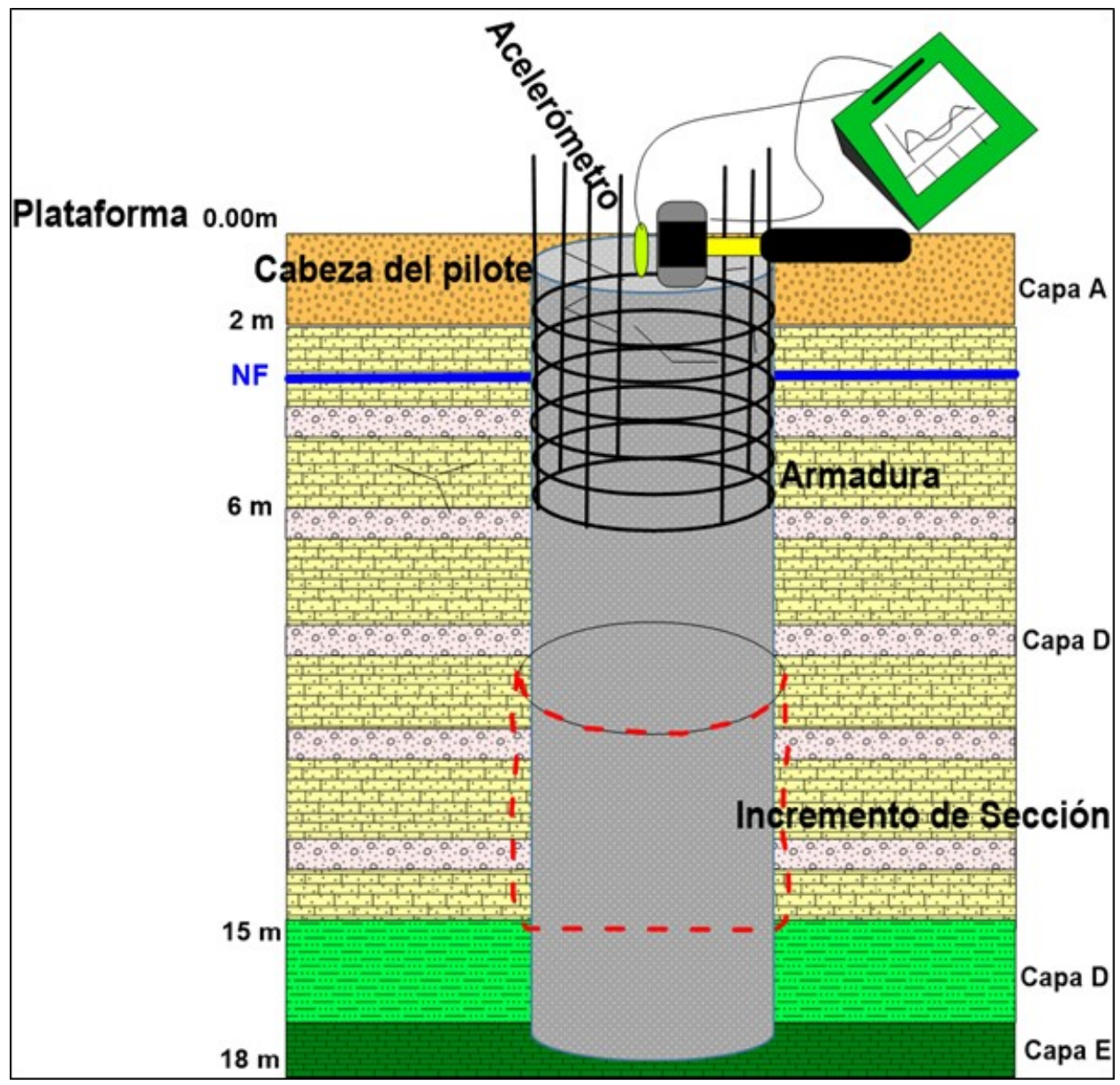

Figura 4. Representación esquemática real de las condiciones de aplicación del ensayo de integridad con el PIT en TP Sector Oasis

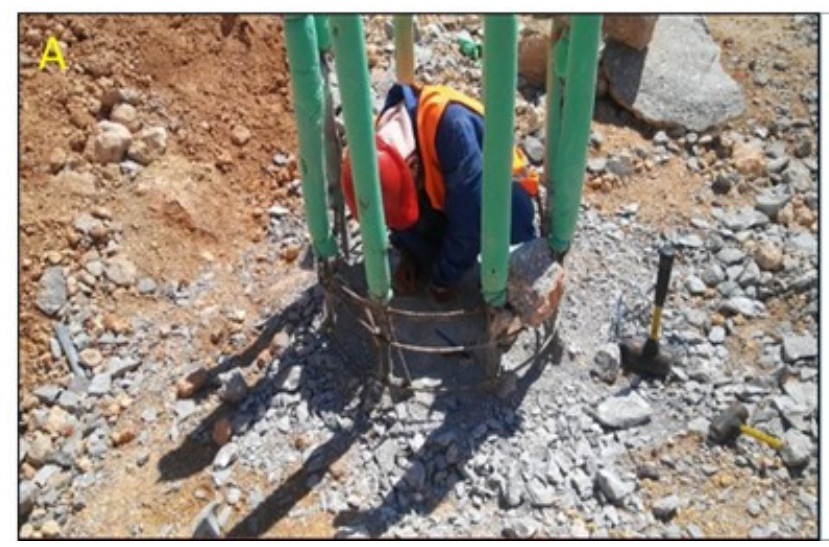

$B$

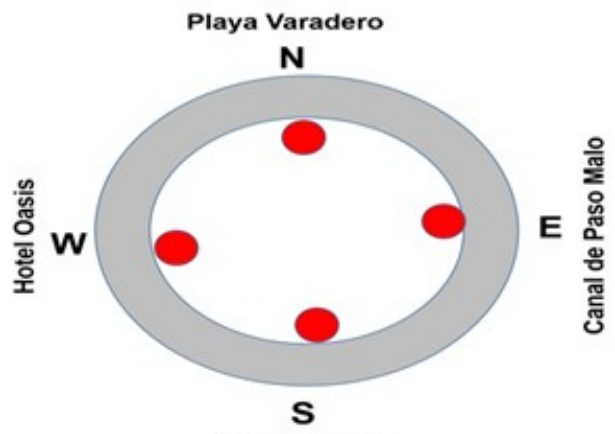

Autopista Sur

Figura 5. Aplicación del ensayo de integridad con el PIT. B. Referencia asumida para indicar en los ensayos las zonas de aplicación del golpe e instalación del acelerómetro. La zona de investigación en el ensayo de PIT se corresponde al área del círculo blanco al borde interior de la armadura del pilote. 
Tratamiento de las curvas en los softwares de procesamiento PIT-S Profile y PIT-W

Los reflectogramas obtenidos en los ensayos de campo fueron almacenados en una memoria externa que se conecta al dispositivo PIT y que luego pudo descargarse como archivos a los softwares PIT-S Profile y PIT-W (Figura 6), con la intención de analizar, con la información disponible el comportamiento de la sección transversal de los pilotes, algunas de las señales tratadas se muestran a continuación acompañadas de una síntesis analítica sobre el criterio final de aceptación de los pilotes (Figuras 7, 8 y 9).

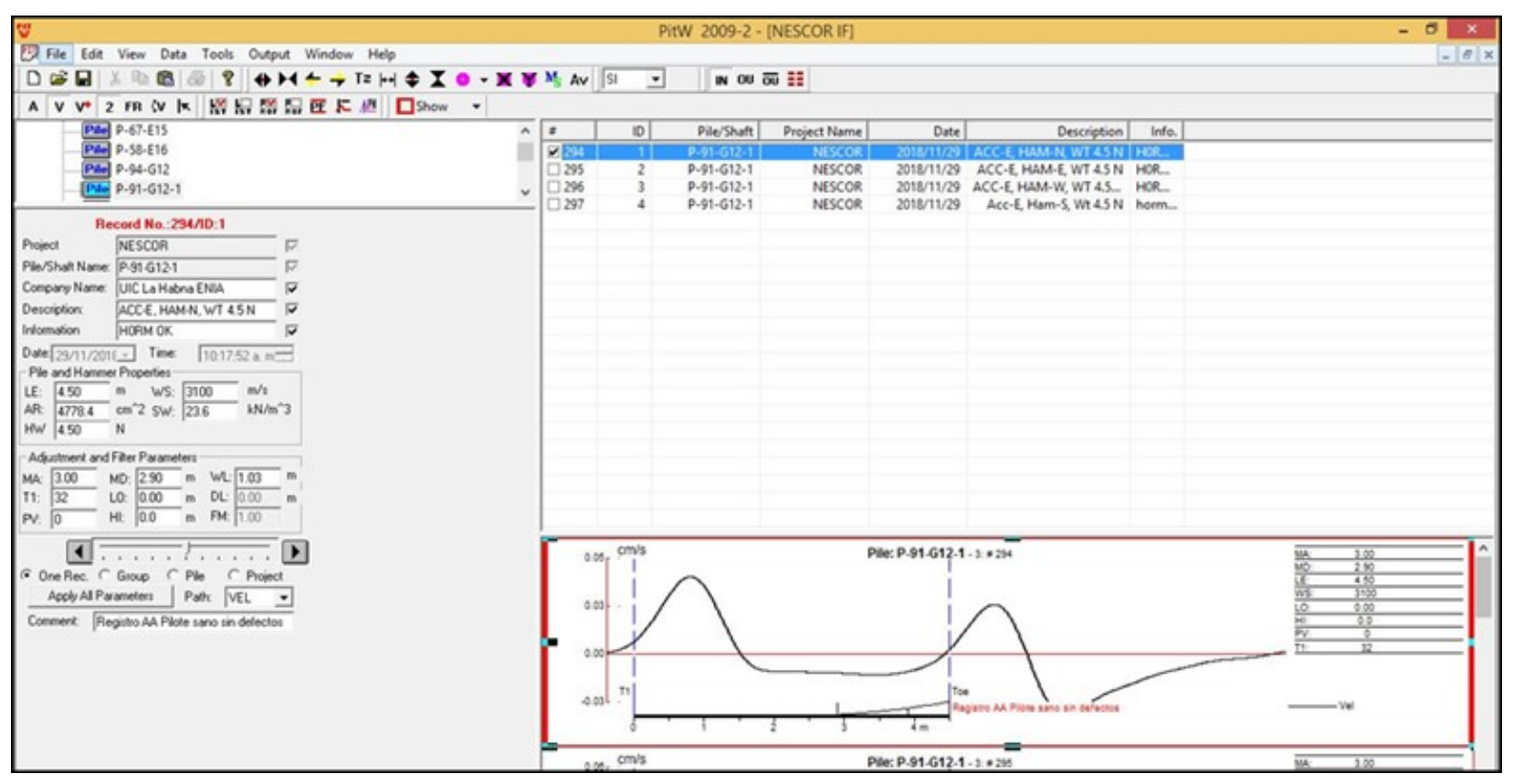

Figura 6. Interfaz del software PIT-W con campos de tratamiento de curvas

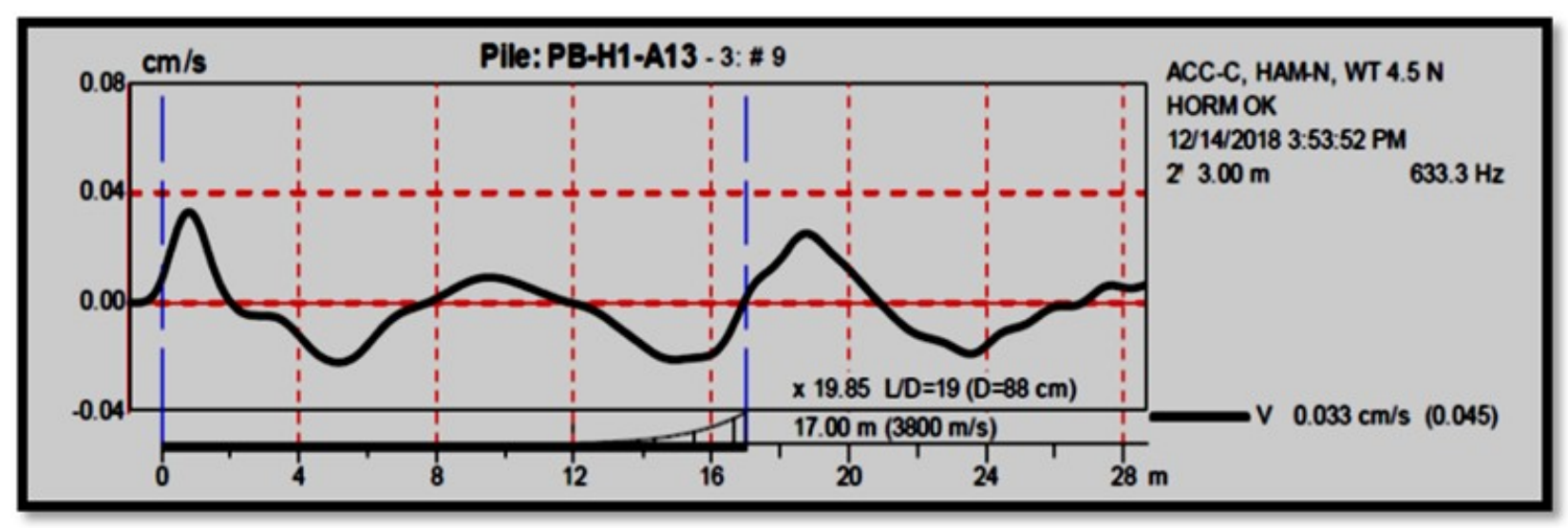

Figura.7. Curva correspondiente al pilote PB-H1-A13, TP Sector Oasis, Varadero

Del registro anterior se puede concluir:

- El pilote tiene continuidad estructural, pues se evidencia de forma clara la reflexión en la punta del pilote a los 17 metros de profundidad. La reflexión de la punta confirma que para la velocidad estimada la longitud del pilote medida coincide con la del reporte Bauer 
- Entre la señal de la cabeza del pilote y la señal de la punta se aprecias cambios (reflexiones) positivos (a 7 metros de profundidad disminución de impedancia) y negativos (aumento de impedancia), dichos cambios representan cambios en la resistencia del hormigón o cambios en la sección del pilote, también los cambios negativos pueden deberse a la interacción suelo-pilote. No tenemos posibilidades de identificar cuál de estos factores es el que predomina.

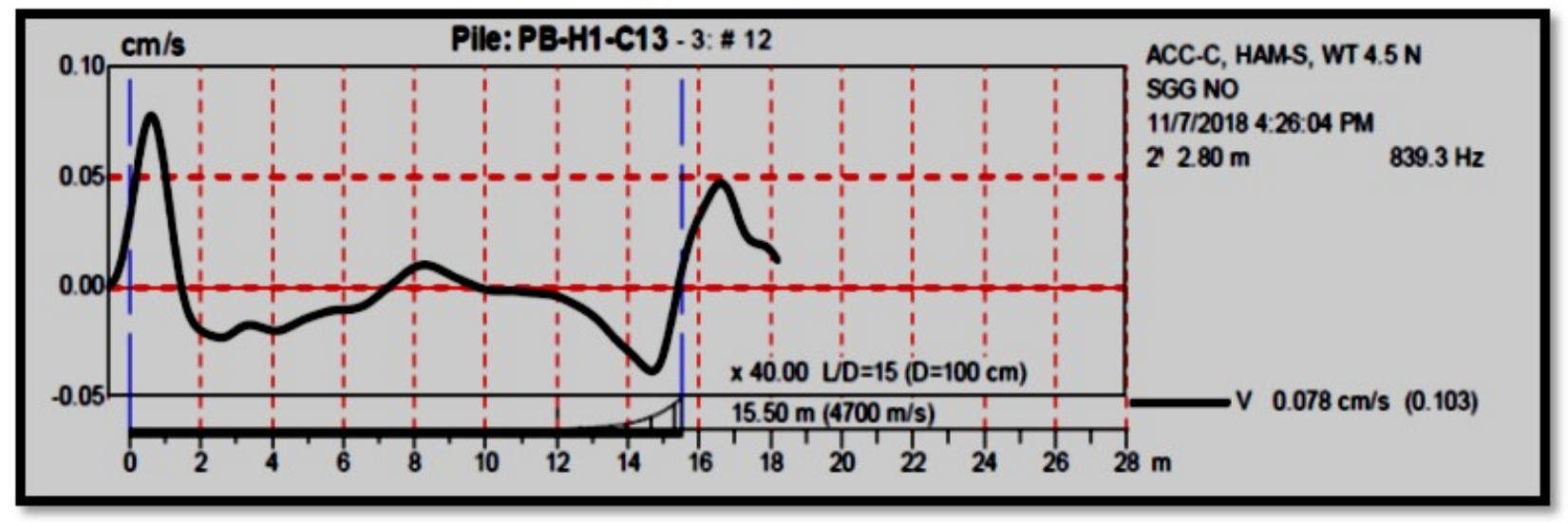

Figura 8. Curva correspondiente al pilote PB-H1-C13, TP Sector Oasis, Varadero

En la imagen anterior se evidencia la curva de un pilote, el cual al igual que la muestra anterior, presenta continuidad estructural, se obtiene la reflexión de la punta del pilote a los 15.5 metros de profundidad. Además, se evidencia cambios de impedancia positivos, este asociado al estrechamiento producto al cambio de sección establecido por proyecto (camisa con diámetro diferente al de diseño). Luego como es común en casi todos los cimientos estudiados, se manifiesta una disminución de impedancia, generalmente en la punta del pilote o cerca de esta, lo que indica en muchos casos el aumento de geometría por el relleno con hormigón de las cavidades de la roca.

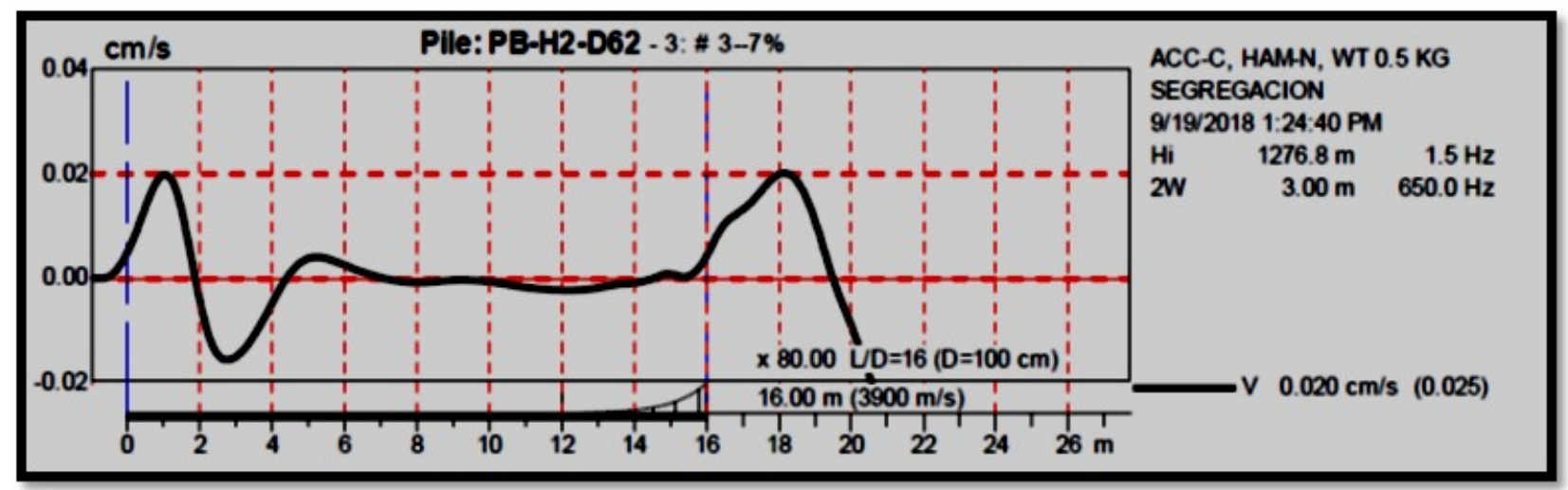

Figura 9. Curva correspondiente al pilote PB-H2-D62, TP Sector Oasis, Varadero

En este caso se puede observar, al igual que todos lo demás, la longitud real del cimiento (16 metros). En el pilote no se aprecia ningún cambio de impedancia positivo lo que indica que a lo largo del cimiento no ocurrió ningún caso de disminución de resistencia o de diminución de la sección. 
De manera general, se da por cumplido el objetivo perseguido en la campaña de investigación consistente en localizar defectos o discontinuidades que pudieran provocar fallos o daños estructurales en los pilotes. La totalidad de los pilotes se consideran aptos para cumplir su función estructural, no estando exentos de presentar daños o defectos provocados durante su construcción pero que, debido al detalle de sobrediseño de estos, fue descartada la posibilidad de fallo ante las solicitaciones que tributarán los edificios a construir.

\section{Conclusiones del Ensayo Sónico de Integridad (PIT)}

1. El ensayo de integridad resulta de suma utilidad en grandes obras por su rapidez de ejecución y factibilidad económica, aun así, al ser una prueba dinámica indirecta, no sustituye la prueba de carga y no se puede determinar capacidad de carga de elementos estructurales.

2. Para analizar la integridad de pilotes, se debe tener en cuenta los problemas encontrados durante su excavación y vertido, debido a que los ensanchamientos de la sección, juntas frías, entre otras patologías constructivas causan rebotes tempranos de la onda que deben ser descartados durante el proceso de interpretación, por lo que una experta interpretación de las curvas de reflexión, debe ir acompañado de la mayor cantidad de información disponible de la construcción de los pilotes.

3. Los ensayos sónicos de integridad representaron también un elemento importante para el control de la calidad considerando que:

- Exploraron los pilotes de manera no invasiva y se detectaron cambios ocultos relacionados con la colocación del hormigón y la construcción del pilote que no aparecen en el reporte Bauer.

- Confirmaron los datos más importantes del reporte Bauer en cuanto a la longitud y continuidad del pilote como elemento estructural.

4. A pesar de la aplicación de los ensayos, los objetivos perseguidos no se cumplieron puesto que la ejecución tardía de estos no pudo impedir el sobrediseño de estos elementos estructurales y un sobreconsumo excesivo en el volumen de hormigón vertido para conformarlo.

5. EL desarrollo del carso en determinados sitios y a determinadas profundidades fue la causa del consumo adicional de hormigón, excusado en el relleno de cavidades y grietas y consecuentemente el factor que originó ensanchamientos de sección en varios pilotes, evidenciados en las crestas negativas obtenidas en los reflectogramas.

Agradecimientos:

A Unidad de Investigaciones Aplicadas para la Construcción UIC La Habana

A Miguel Ángel Maestre Mesa

A Manuel Aguilar Balbuena 


\section{Referencias Bibliográficas}

ASTM D5882-00. (s.f.). “'ASTM D 5882, 2000. Standard test method for low strain integrity testing of piles. American Society for Testing and Materials. ".

Colectivo de Autores Pile Dynamics Inc. (2014). Manual PIT.

Fundaciones, L. d. (s.f.). Protocolo de ensayo de integridad de pilotes.

Maestre Mesa, M Á., Rodríguez Cárdenas, H. (2019). Informe Final del grupo de Patología y Restauración ENIA UIC-La Habana. Aplicación de ensayos PIT a pilotes hormigonados in situ en TP-Sector Oasis. Varadero, Cuba.

Santos, Y. (2016). Aplicación de la Prueba de Integridad de Pilotes en puentes de Cayo. Santa Clara. 